\title{
Identification and validation of a five-gene prognostic signature for hepatocellular carcinoma
}

\author{
Huibin Yang ${ }^{1}$, Junyu Huo ${ }^{2}$ and Xin $\mathrm{Li}^{2^{*}}$ (1)
}

\begin{abstract}
Background: ARID1A is a commonly mutated tumor suppressor gene found in all human cancer types, but its clinical significance, oncogenic functions, and relevant mechanisms in hepatocellular carcinoma (HCC) are not well understood.

Objective: We aimed to improving the prognosis risk classification of HCC from the perspective of ARID1A mutations.

Materials and methods: We examined the interaction between ARID1A mutations and the overall survival via Kaplan-Meier survival analysis. We used gene set enrichment analysis (GSEA) to elucidate the influence of ARID1A mutations on signaling pathways. A prognostic model was constructed using LASSO and multivariate Cox regression analyses. A receiver operating characteristic (ROC) curve was used to estimate the performance and accuracy of the model.

Results: HCC patients with ARID1A mutations presented poor prognosis. By GSEA, we showed that genes upregulated by reactive oxygen species (ROS) and regulated by MYC were positively correlated with ARID1A mutations. A prognostic signature consisting of 5 genes (SRXN1, LDHA, TFDP1, PPM1G, and EIF2S1) was constructed in our research. The signature showed good performance in predicting overall survival (OS) for HCC patients by internal and external validation.
\end{abstract}

Conclusion: Our research proposed a novel and robust approach for the prognostic risk classification of HCC patients, and this approach may provide new insights to improve the treatment strategy of HCC.

Keywords: Hepatocellular carcinoma, ARID1A mutations, Prognostic, Signature

\section{Background}

A recent study on the next-generation sequencing of 669 patients with 24 types of cancers showed that the average mutation rate of the SWI/SNF gene was $19 \%$, which was only lower than the mutation rate of TP53 (26\%); therefore, the SWI/SNF gene is ranked second

\footnotetext{
*Correspondence: joemarton@163.com

${ }^{2}$ Department of Hepatobiliary and Pancreatic Surgery, The Affiliated Qingdao Municipal Hospital of Qingdao University, No.1 Jiaozhou Road, Shibei District, Qingdao City 266011, Shandong Province, China

Full list of author information is available at the end of the article
}

among all mutated genes, making the SWI/SNF complex and its mediated nucleosome chromatin remodeling the focus of cancer research [1]. The ARID1A (AT-rich binding domain 1A) gene, also known as BAF250a, is the core subunit of the chromatin remodeling complex SWI/SNF $[2,3]$ and may help to control the specificity exhibited by SWI/SNF in the whole genome nucleosome remodeling and affect the composition and catalytic ability of the whole complex [4]. The chromatin remodeling complex participates in the process of DNA replication, transcription, and repair, participates in the regulation of

(c) The Author(s). 2021 Open Access This article is licensed under a Creative Commons Attribution 4.0 International License, which permits use, sharing, adaptation, distribution and reproduction in any medium or format, as long as you give appropriate credit to the original author(s) and the source, provide a link to the Creative Commons licence, and indicate if changes were made. The images or other third party material in this article are included in the article's Creative Commons licence, unless indicated otherwise in a credit line to the material. If material is not included in the article's Creative Commons licence and your intended use is not permitted by statutory regulation or exceeds the permitted use, you will need to obtain permission directly from the copyright holder. To view a copy of this licence, visit http://creativecommons.org/licenses/by/4.0/. The Creative Commons Public Domain Dedication waiver (http://creativecommons.org/publicdomain/zero/1.0/) applies to the data made available in this article, unless otherwise stated in a credit line to the data. 
the PI3K/Akt signaling pathway, and carries out chromatin remodeling with the histone covalent modification complex, which is related to the inhibition of tumorigenesis and development $[5,6]$.

Recently, research on the function and mechanism of ARID1A in tumors have made important progress. ARID1A inhibits cell proliferation by regulating the cell cycle, induces the expression of P21 factor, promotes its binding to the cyclin CDK2/CDK4 complex, and inhibits its activity so that the cell cycle is stagnated in the G1 phase. ARID1A expression is downregulated in other stages, except in the G0 phase, and is almost completely deficient in cells with exuberant cell division [3]. ARID1A deficiency can change the gene expression profile of embryonic stem cells, increase the expression of the cell development-related genes Gata4, Ga-ta6, Tnt2, and Myl3, and decrease the expression of stem cell selfrenewal genes, which further confirms the importance of ARID1A in stem cell maintenance and differentiation [6]. The ARID1A gene was knocked out in leukemic cells, and it was found that apoptosis mediated by fas was inhibited [7]. ARID1A can also promote apoptosis by regulating the target genes $\mathrm{Bcl}-2$ and cyclin D1 [7]. Therefore, ARID1A, as an important tumor suppressor gene, may remarkably affect tumor occurrence and development.

Huang et al. [8] used exon sequencing technology to detect somatic mutations in 110 patients with portal vein tumor thrombi (PVTTs) and patients with HBVpositive hepatocellular carcinoma (HCC). They found that the mutation of the ARID1A gene was the most important, and the mutation rate of the ARID1A gene was $13 \%(14 / 110)$, indicating that it remarkably affected HCC occurrence and development. Increasing evidences suggested that decreased expression of ARID1A in HCC patients was associated with poor prognosis and could promoted metastasis of HCC $[9,10]$, and ARID1A could also regulate response to anti-angiogenic therapy in advanced $\mathrm{HCC}$ [11], implying that ARID1A may represent a promising candidate therapeutic target for HCC. However, the molecular mechanism of ARID1A in HCC remains to be clarified.

The study exploring the potential molecular mechanism for ARID1A mutation with HCC via gene set enrichment analysis (GSEA). We proposed a five-gene signature to evaluate HCC prognosis and carried out internal and external verification, which will guide the clinical management of HCC.

\section{Materials and methods}

\section{Data collection}

We downloaded the mRNA expression profile of 374 HCC samples from The Cancer Genome Atlas (TCGALIHC) website (https://portal.gdc.cancer.gov/projects/
TCGA-LIHC). Corresponding clinical information was available on the UCSC Xena website (https://tcga-xenahub.s3.us-east-1.amazonaws.com/latest/TCGA.LIHC. sampleMap\%2FLIHC_clinicalMatrix; Full metadata). We acquired the sample list of ARID1A alterations from the cBioPortal website (https://www.cbioportal.org/). We obtained sequence data, somatic mutation data, together with the corresponding clinical data for 228 HCC samples from the International Cancer Genomics Consortium (ICGC-LIRI-JP, https://dcc.icgc.org/projects/LIRIJP). TCGA and ICGC were all based on Illumina HiSeq platform. This study meets the publication requirements of the TCGA and ICGC. The detailed clinical information of all included samples as shown in Table 1. The data of this study were from a public database, so they do not need to be approved by the local ethics committee.

\section{Gene set enrichment analysis (GSEA)}

To explore the underlying molecular mechanism regarding ARID1A mutations in HCC, we performed gene set enrichment analysis on the samples with $(n=36)$ and without $(n=338)$ ARID1A mutation. To identify the pathways that were significantly enriched between patients with and without ARID1A mutations, we selected an annotated gene set file (h.all.v7.1.symbols.gmt) as the reference. The threshold was confirmed as $\operatorname{NOM} p$ value $<0.05$; FDR $q$ value $<0.25$. GSEA software (http://www. broadinstitute.org/gsea) was applied to GSEA.

\section{Construction of a five-gene prognostic signature}

The "caret" package in $\mathrm{R}$ software assisted in classifying the 343 HCC patients who survived more than one month from the TCGA-LIHC project into the training cohort $(n=172)$ and the testing cohort $(n=171)$ of equal sample size in a random manner. No statistically significant difference in the clinico-pathological parameter was detected between the training and validation set by the chi-square test (supplement material 2), which meaned means that we excluded the effect of clinical factors on the prognosis of patients in both cohorts. We extracted gene sets significantly upregulated in ARID1A mutation samples, and univariate Cox regression analysis assisted in identifying prognostic genes in the training cohort. Then, important mRNAs were confirmed from the prognostic genes via the least absolute shrinkage and selection operator (LASSO) model. Multivariate Cox proportional regression analysis served to establish a risk score model with the identified mRNAs. The KaplanMeier (KM) analysis together with the receiver operating characteristic (ROC) curve assisted in evaluating the clinical value exhibited by the risk score. Univariate and multivariate Cox regression helped analyze the factors related to prognosis in $\mathrm{HCC}$ patients. 
Table 1 The detailed clinical information of all included samples

\begin{tabular}{lll}
\hline & TCGA & ICGC \\
\hline Survival status & 224 & 187 \\
Alive & 119 & 41 \\
Dead & & 88 \\
Age & 127 & 140 \\
$\leq 65$ & 216 & 167 \\
$>65$ & & 61 \\
Gender & 233 & 110 \\
Male & 110 \\
Female & & 140 \\
Stage & 238 & 88 \\
I-II & 83 & 29 \\
III-IV & & 199
\end{tabular}

Grade

G1 53

G2 161

$\begin{array}{ll}\text { G3-4 } & 124\end{array}$

$\mathrm{BMl}$

$\leq 25 \quad 163$

$>25 \quad 153$

AFP

$\begin{array}{ll}\leq 300 \mathrm{ng} / \mathrm{ml} & 197\end{array}$

$>300 \mathrm{ng} / \mathrm{ml} \quad 62$

New tumor event after initiate treatment

Yes

No

162

Cancer status

With tumor

Tumor free

179

Vascular tumor cell type

None

188

Micro and marco

Race

White

Others

164

Additional_pharmaceutical_therapy

No

Ye

29

Residual_tumor

R0 
Table 1 The detailed clinical information of all included samples (Continued)

\begin{tabular}{ccc}
\hline & TCGA & ICGC \\
\hline R1-2 & ARID1A & \\
& & \\
Mutation & 25 & 23 \\
Wild & 306 & 203 \\
& & 306 \\
\hline
\end{tabular}

\section{The five-gene prognostic signature validation}

After the construction of the prognostic signature, we used the testing cohort $(n=171)$ together with the entire TCGA cohort $(n=343)$ to verify the accuracy exhibited by the prognostic risk model. To ensure the stability of the validation results, patients from TCGA were grouped according to their clinical features, and survival analysis was carried out by subgroup. An independent dataset (ICGC, $n=228$ ) was employed for external validation for its effectiveness in overall survival (OS) prediction specific to HCC patients.

\section{Results}

\section{ARID1A alteration associated with increased gene mutation count}

The mutation frequency of ARID1A in TCGA datasets was $9 \%$. Truncating mutation was the main mutation type, followed by missense mutation, and amplification was the rarest (Fig. 1a). The gene mutation count of the ARID1A-altered group was higher than that in the unaltered group, which was statistically significant (Fig. 1b). The fraction of ARID1A-altered exhibited positive correlation with gene mutation count (Fig. 1c). Interestingly, the top $25 \%$ of samples with the highest gene mutation count were assigned into the genomic unstable (GU) group, whereas the bottom 25\% with the lowest gene mutation count were classified as genomic stable (GS) group [12], the two groups differed greatly in overall survival (OS) (Fig. 1d). The somatic mutation data (TCGA.LIHC.varscan.somatic.maf) were downloaded from TCGA (https://tcga-data.nci.nih.gov/tcga/). The subsequent workflow chart of this research is shown in supplement file 1 .

\section{Identification of gene sets enriched in ARID1A-mutated HCC samples}

By comparing the OS of HCC patients with ARID1A mutations and those without ARID1A mutations, we found that the OS of those with ARID1A mutations was obviously lower than that of those without ARID1A mutations (Fig. 2a). We conducted GSEA on the altered group and unaltered group to investigate the underlying molecular mechanism. After screening, three gene sets were considered to be positively correlated with ARID1A mutations (Fig. 2b, Table 2). A total of 288 genes were extracted for the subsequent analyses.

\section{Construction of a five-gene prognostic signature based on the training cohort}

As revealed by univariate Cox regression analysis, 28 genes exhibited an association with patient OS $(p<$

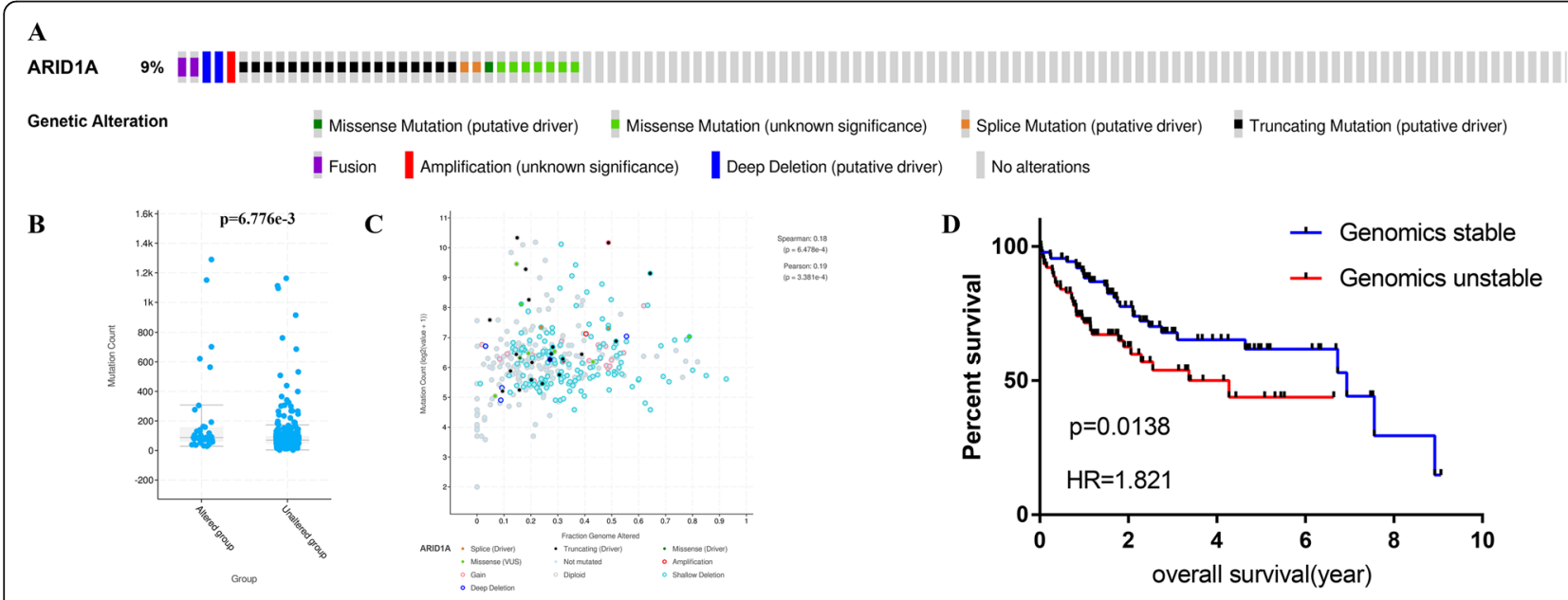

Fig. 1 The landscape of ARID1A alteration in TCGA. a The mutation type of ARID1A. b, $\mathbf{c}$ The relationship between the fraction of ARID1A-altered with gene mutation count. d The Kaplan-Meier survival analysis regarding genomic unstable (GU) group and genomic stable (GS) group 


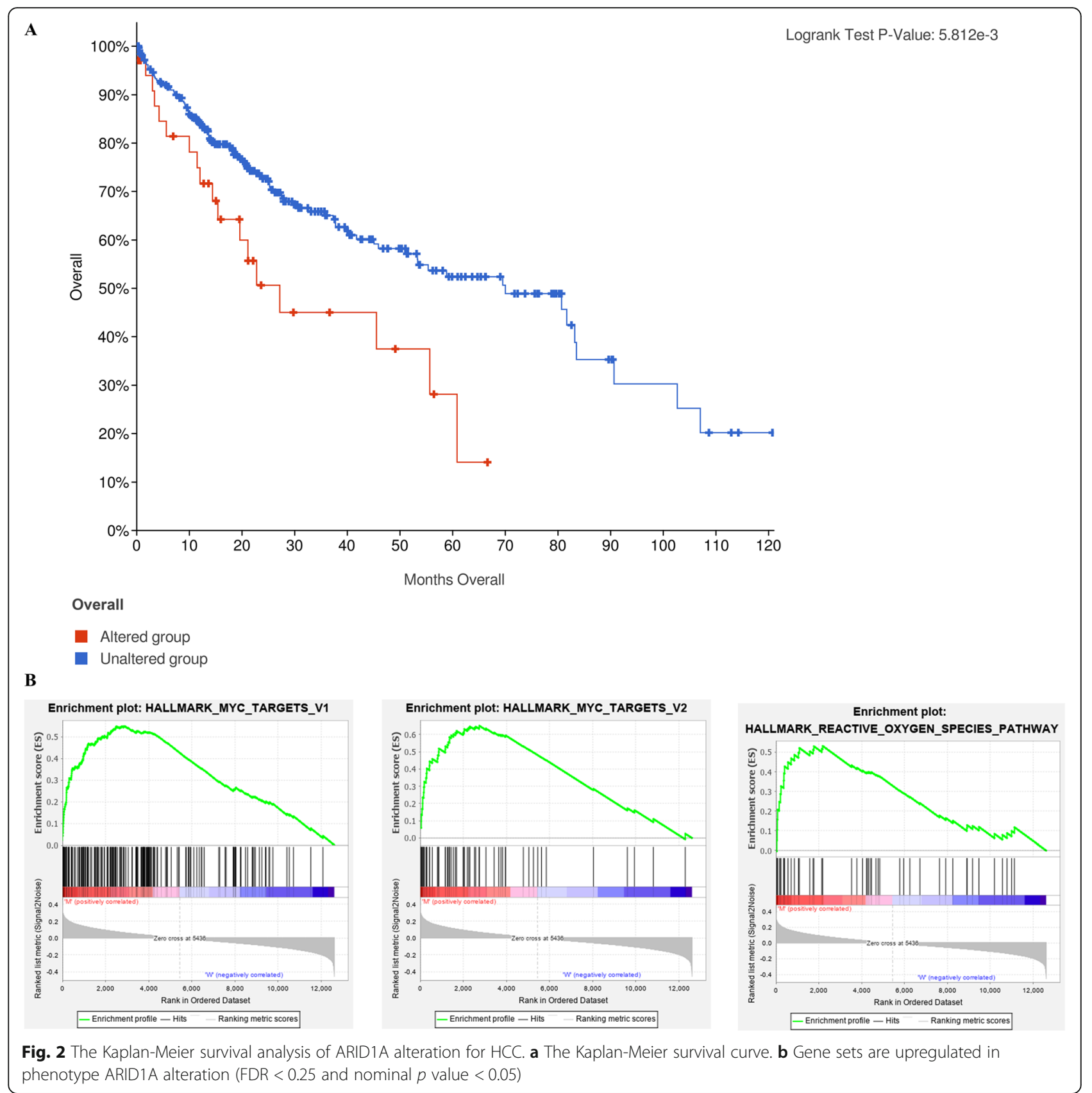

0.001 , Table 3). Then, genes that may show a close relation to other genes were excluded through LASSO regression analysis. The 8 genes selected from LASSO regression analysis were retained for multivariate Cox regression analysis. These prognostic gene expression markers were linearly combined with the regression coefficient $(\beta)$ in the multivariate Cox regression analysis to construct a risk score model. The risk score was calculated as follows: SRXN1"0.03974+ LDHA*0.003247+ TFDP1*0.022758+ PPM1G*0.037018+ EIF2S1*0.063182.

Table 2 Gene set enrichment analysis for ARID1A alteration HCC samples

\begin{tabular}{|c|c|c|c|c|}
\hline Gene sets & ES & NES & NOM p-val & FDR q-val \\
\hline HALLMARK_MYC_TARGETS_V2 & 0.650381 & 1.851576 & 0.015521 & 0.19292 \\
\hline HALLMARK_REACTIVE_OXYGEN_SPECIES_PATHWAY & 0.53105 & 1.770816 & 0.024176 & 0.187634 \\
\hline HALLMARK_MYC_TARGETS_V1 & 0.549157 & 1.754343 & 0.036403 & 0.14646 \\
\hline
\end{tabular}


Table 3 The prognostic gene list

\begin{tabular}{lllll}
\hline Gene name & HR & HR.95L & HR.95H & $\boldsymbol{p}$ value \\
\hline TXNRD1 & 1.014623 & 1.008958 & 1.020319 & $3.73 \mathrm{E}-07$ \\
TFDP1 & 1.03243 & 1.019555 & 1.045468 & $6.21 \mathrm{E}-07$ \\
SRXN1 & 1.048058 & 1.028743 & 1.067735 & $7.58 \mathrm{E}-07$ \\
EIF2S1 & 1.222953 & 1.125922 & 1.328346 & $1.82 \mathrm{E}-06$ \\
PSMD1 & 1.096507 & 1.055521 & 1.139086 & $2.14 \mathrm{E}-06$ \\
PPM1G & 1.049258 & 1.027294 & 1.071692 & $8.40 \mathrm{E}-06$ \\
GCLM & 1.031107 & 1.017293 & 1.045107 & $8.53 \mathrm{E}-06$ \\
KPNA2 & 1.01951 & 1.010513 & 1.028586 & $1.93 \mathrm{E}-05$ \\
LDHA & 1.005669 & 1.003064 & 1.008281 & $1.94 \mathrm{E}-05$ \\
MCM6 & 1.06796 & 1.036161 & 1.100735 & $2.02 \mathrm{E}-05$ \\
SERBP1 & 1.050061 & 1.026388 & 1.074281 & $2.69 \mathrm{E}-05$ \\
RRM1 & 1.056424 & 1.029482 & 1.084072 & $3.12 \mathrm{E}-05$ \\
PRDX1 & 1.002777 & 1.001431 & 1.004125 & $5.23 \mathrm{E}-05$ \\
PSMB2 & 1.041963 & 1.021234 & 1.063114 & $6.09 \mathrm{E}-05$ \\
CCT4 & 1.028998 & 1.014373 & 1.043834 & $9.08 \mathrm{E}-05$ \\
MCM2 & 1.060914 & 1.029698 & 1.093077 & 0.000104 \\
USP1 & 1.096038 & 1.045936 & 1.148539 & 0.000122 \\
CCT5 & 1.024885 & 1.011956 & 1.03798 & 0.000148 \\
GSR & 1.022544 & 1.010775 & 1.034451 & 0.00016 \\
ETF1 & 1.070413 & 1.033074 & 1.109102 & 0.000173 \\
XPO1 & 1.061489 & 1.027121 & 1.097007 & 0.00038 \\
PSMD14 & 1.060731 & 1.026673 & 1.095918 & 0.000399 \\
CAD & 1.124055 & 1.053586 & 1.199237 & 0.0004 \\
PSMA1 & 1.029786 & 1.013009 & 1.046842 & 0.000462 \\
CUL1 & 1.070192 & 1.029964 & 1.111991 & 0.00052 \\
NCBP1 & 1.234655 & 1.094769 & 1.392415 & 0.000591 \\
CCT7 & 1.017927 & 1.007499 & 1.028462 & 0.00072 \\
CCT3 & 1.007581 & 1.003075 & 1.012108 & 0.000957 \\
\hline & & & & \\
\hline
\end{tabular}

The median risk score (0.85) was taken as the threshold for dividing patients in the training set into a group with a high risk and a group with a low risk. As shown in the KM survival curve, the prognosis of patients in the group with a high risk presented statistical significance (Fig. 3a). The area under the ROC curve (AUC) values for the risk score in predicting 1-year, 2-year, 3-year, 4-year, and 5-year OS were 0.861, $0.733,0.750,0.764$, and 0.726 , respectively (Fig. 3b), indicating that this prognostic signature has a better predictive power for HCC patient survival.

\section{Internal validation of the five-gene prognostic signature in the testing cohort}

The risk score regarding the 171 patients in the testing set was calculated further. We divided them into a group with a high risk and a group with a low risk considering the same cutoff point (0.85) in the training set. As shown in Fig. 3c, patients in the high-risk group presented an obviously lower OS than those in the lowrisk group. The risk score provided the highest 1-year AUC (0.813), which was still considered to represent a high predictive efficacy (Fig. 3d).

\section{Validation of the five-gene prognostic signature in the TCGA cohort}

To validate the accuracy exhibited by the risk model, we analyzed the model in the entire TCGA cohort. Consistent with the foregoing results, patients in the high-risk group had a substantially worse outcome than patients in the low-risk group $(P<0.001)$ (Fig. 3e). The AUC ranged from 0.692 to 0.838 , which showed an excellent predictive capability of the model (Fig. 3f).

\section{Subgroup analysis of the five-gene prognostic signature}

From the results of both univariate and multivariate Cox regression analyses, the risk score was found to be an independent prognostic indicator (Fig. 4a, b), and we validated the applicability of the prognostic value given by our model to other clinical factors. We divided the patients into 24 subgroups based on their clinicopathologic characteristics, and next, each subgroup was further separated into a group with a high risk and a group with a low risk relying on the 5-gene signature. Kaplan-Meier analysis assisted in estimating the OS of different subgroups, and a log-rank test helped confirm the prognostic difference. As revealed, the group with a low risk saw a smaller number of mortalities relative to the group with a high risk in each clinical subgroup (Fig. 4c), which confirmed the robustness of our signature.

\section{External validation of the five-gene prognostic signature in the ICGC cohort}

To further evaluate whether the prognostic model was reliable, an external dataset from the ICGC database was adopted. The risk score of the 228 patients in the ICGC cohort was taken as a criterion to divide them into a group with a high risk and a group with a low risk, which was similar to the step regarding the TCGA cohort. In line with expectations, patients in the high-risk group exhibited poorer OS $(P<0.001$; Fig. 5a). The AUCs of the risk score in predicting 1-year, 2-year, 3year, 4-year, and 5-year OS were 0.746, 0.750, 0.778, 0.778 , and 0.778 , respectively (Fig. $5 \mathrm{~b}$ ). The risk score distribution plot and survival status plot demonstrated that the risk for death increased as the risk score increased (Fig. 5c, d). In line with univariate and multivariate analyses, the risk score could help to predict the prognosis of patients $(P<0.001$, Fig. 5 e, f). It is worth mentioning that the prognostic model was also suitable for patients with different clinical features (Fig. 6a-d). These results demonstrated that successful external validation of our model was achieved. 
A

Risk + High risk — Low risk

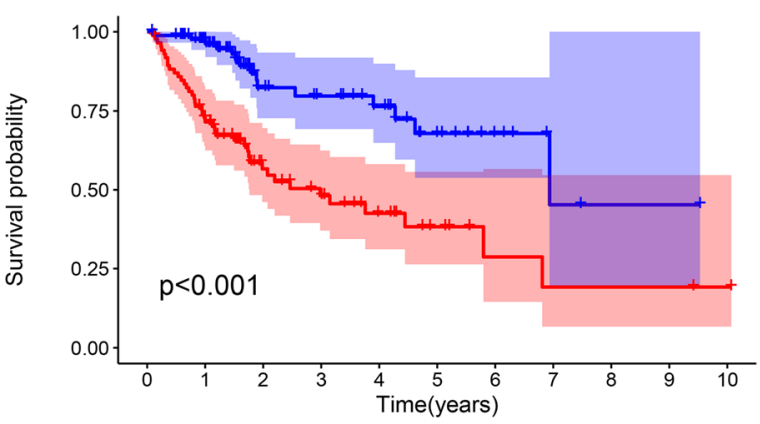

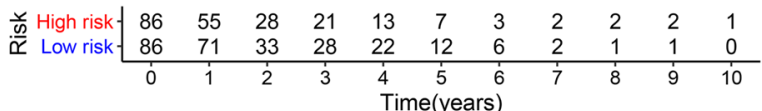

training cohort

C

Risk — High risk — Low risk

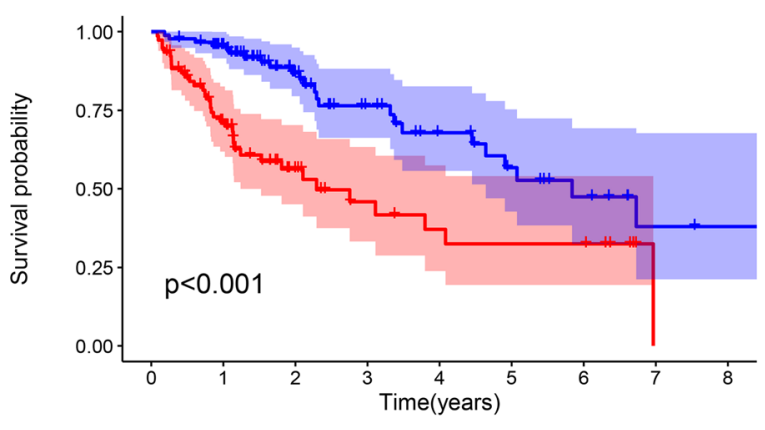

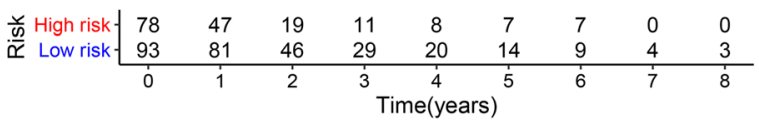

testing cohort

E

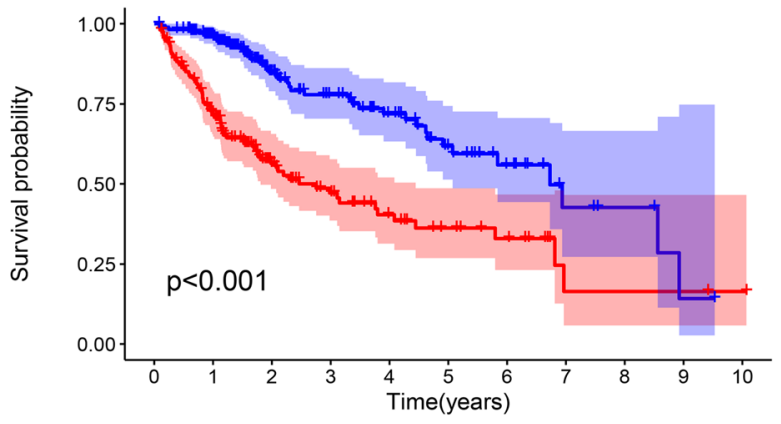

B

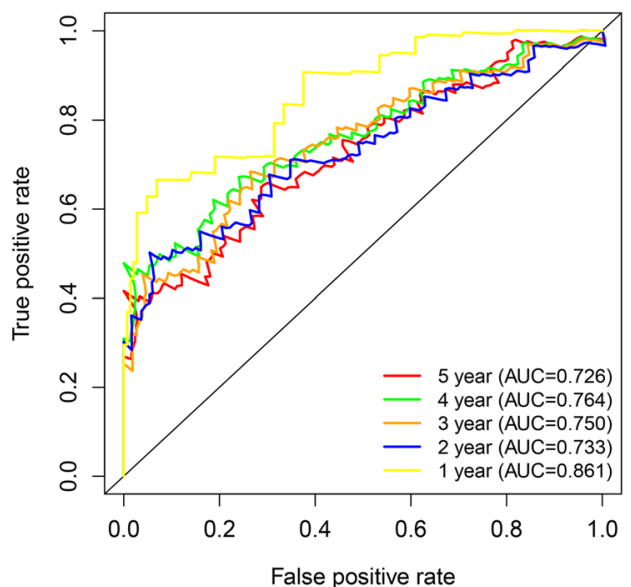

D

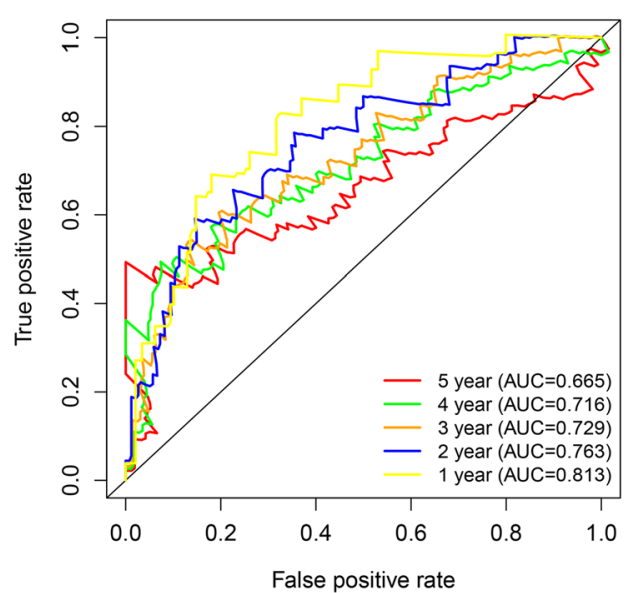

riskScore

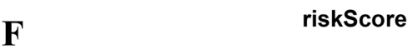

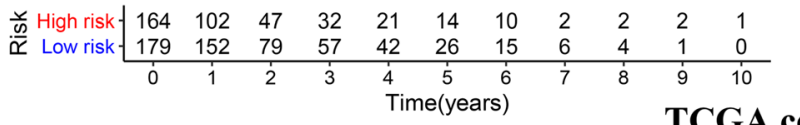

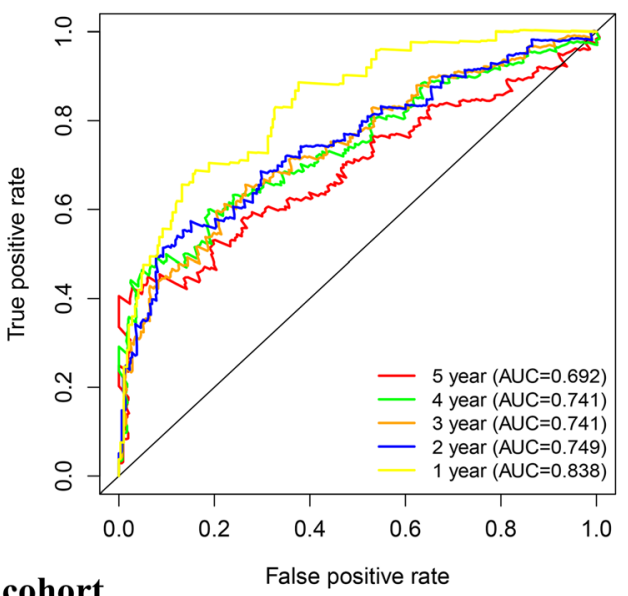

Fig. 3 (See legend on next page.) 
(See figure on previous page.)

Fig. 3 The establishment of the 5-gene signature. a Kaplan-Meier survival analysis and time-dependent ROC analysis of predicting overall survival for patients in training cohort used by risk score. $\mathbf{b}$ Kaplan-Meier survival analysis and time-dependent ROC analysis of predicting overall survival for patients in testing cohort used by risk score. c Kaplan-Meier survival analysis and time-dependent ROC analysis of predicting overall survival for patients in whole TCGA cohort used by risk score

\section{The prognostic model showed superior to TNM stage}

The TNM stage was still the most common instrument predict the prognosis of HCC in clinical practice currently. By comparing the AUC values, we found that the risk score with better performance, especially in predicting the long-term survival of HCC (Fig. 7).

\section{Discussion}

HCC is a serious threat to human health

Hepatocellular carcinoma (HCC) accounts for 85-90\% of primary liver cancers, is the 2nd leading cause of cancer-related death, is the 4th most common cancer worldwide, and has the 6th highest incidence [13, 14]. HCC is usually characterized by occult onset, early

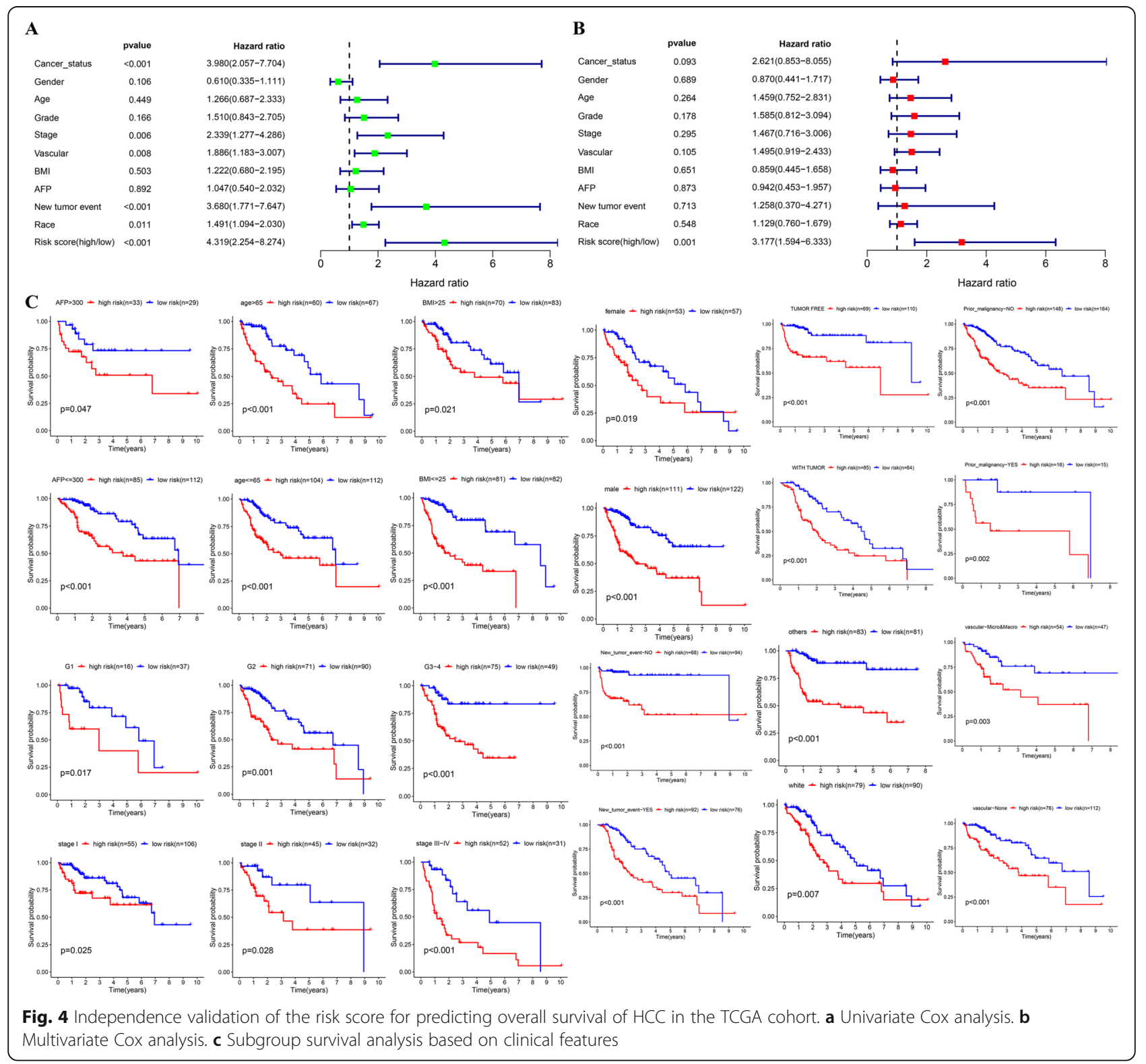




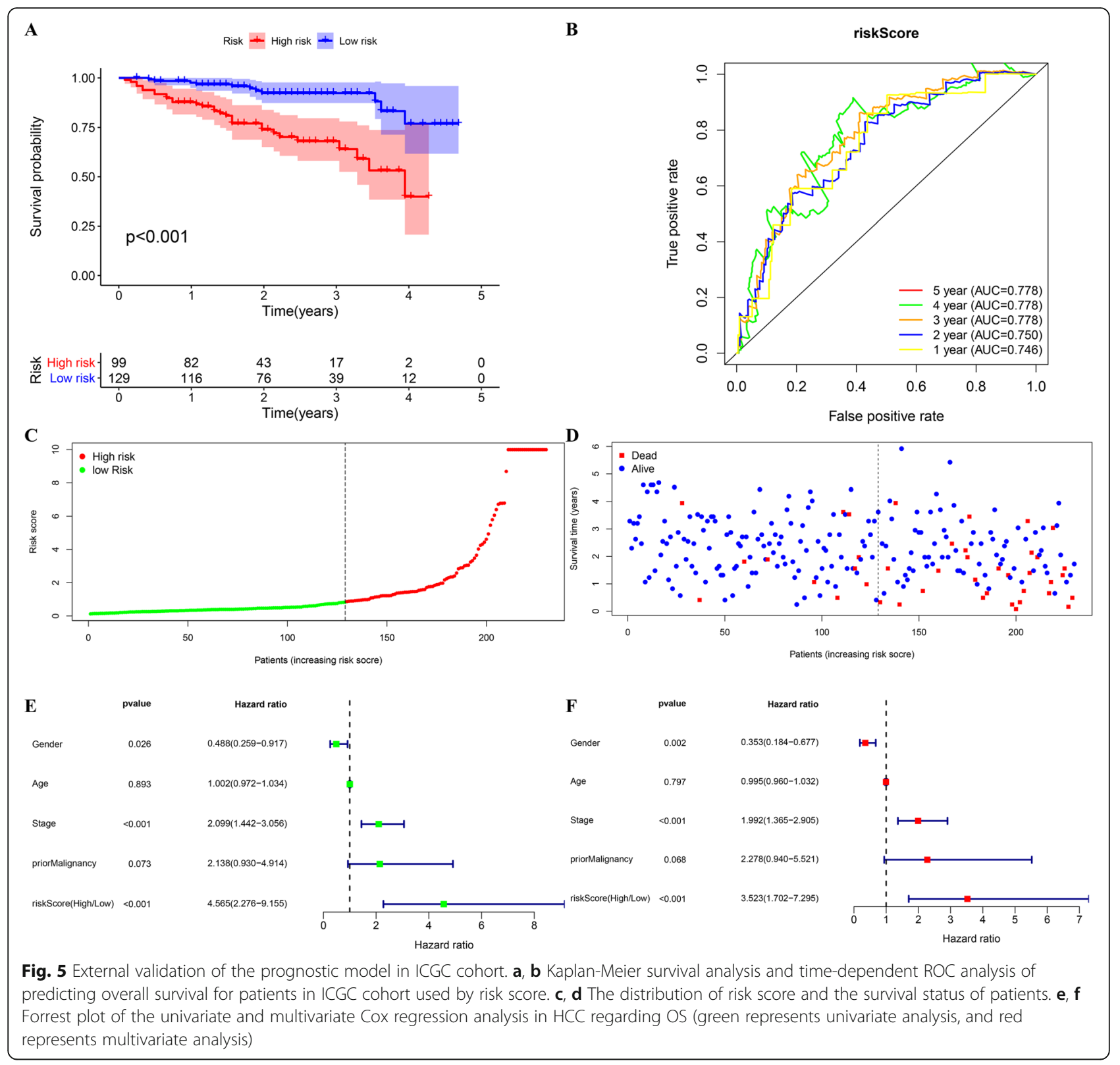

asymptomatic manifestation, and rapid development. Most HCC patients have progressed to the middle or advanced stage upon diagnosis [15]. Therefore, it is of great significance to prolong the survival time and improve the quality of life of patients if we can monitor the curative effect and prognosis of HCC in real time.

\section{The heterogeneity of $\mathrm{HCC}$ is a challenge}

HCC is a kind of malignant tumor with high heterogeneity [16]. Even in patients in the same clinical stage, the molecular characterization of tumor cells is still very different. According to the molecular classification, different types of patients have different clinical efficacies and prognoses [17]. In recent years, as high-throughput sequencing has been developed rapidly, exploring biomarkers of immunotherapy and molecular targeted therapy based on molecular typing can accurately identify the population of patients who would benefit and predict the efficacy and prognosis of drugs, which has become a popular research topic [18].

\section{ARID1A mutation in HCC remains to be elucidated}

In HCC, epigenetic modifications often change. ARID1A, an SWI/SNF chromatin-remodeling gene, usually is mutated in cancer and is considered to be capable of suppressing tumors $[19,20]$. Sun et al. [21] revealed the context-specific function possessed by ARID1A, an SWI/SNF component, in liver cancer. Increased ARID1A 

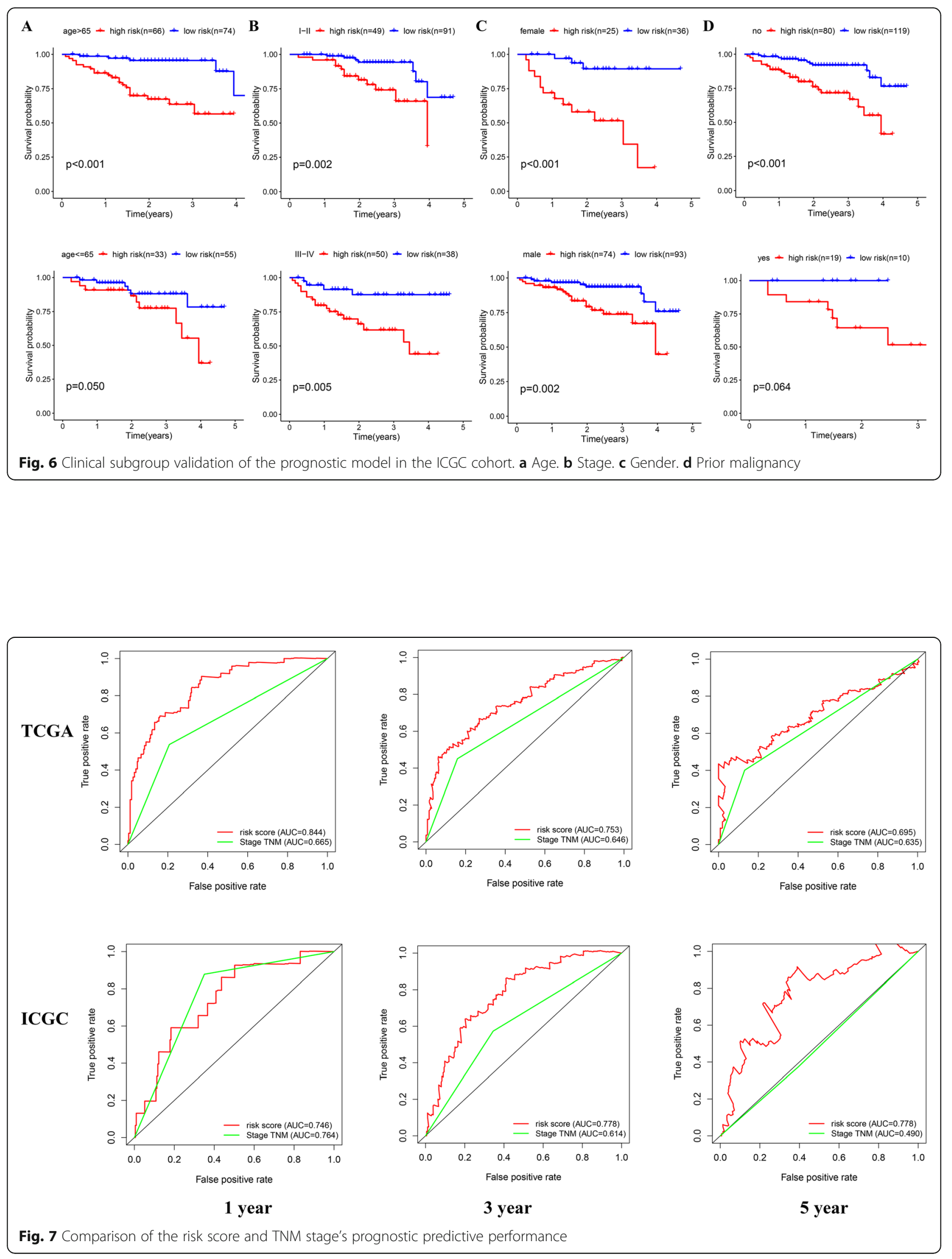
facilitates tumor initiation via oxidative stress mediated by CYP450, and decreased ARID1A in established tumors strengthens metastasis because inhibitory factors present lower expression. An increasing number of studies have reported that ARID1A is associated with the OS of liver cancer patients [22, 23], but the specific mechanism is still unclear.

\section{ARID1A mutation may lead to the upregulation of ROS- and MYC-related genes}

Our research found that HCC patients with ARID1A mutations showed substantially poor prognoses. By GSEA, we found that the genes related to reactive oxygen species (ROS) and MYC were positively correlated with ARID1A mutations. The MYC oncogene leads to many human cancers [24]. Studies that have been conducted recently regarding the expression and function of MYC have provided new insight into MYC therapy [25]. For example, drug-like molecules could inhibit bromodomain-induced MYC activation, accordingly inhibiting tumors in vivo [26]. It is also possible to suppress tumor growth through pharmacologically uncoupling bioenergetic pathways that involve glutamine or glucose metabolism from cellular biomass accumulation induced by MYC [27]. MYC can also be prevented from evolving into cancer by targeting Myc-Max dimerization or Myc-induced microRNA expression [24]. It has long been believed that unstable reactive oxygen species (ROS) promote cancer production by causing DNA damage and activating oncogenes $[28,29]$. However, to date, there has been no definite answer to whether the expression of these genes is related to the prognosis of HCC.

\section{The prognostic signature containing 5 genes showed good performance}

We extracted the genes related to reactive oxygen species (ROS) and MYC from gene expression profiles and analyzed their prognostic value for HCC. A prognostic signature consisting of 5 genes (SRXN1, LDHA, TFDP1, PPM1G, and EIF2S1) was constructed in our research. After the completion of the model construction, we carried out four levels of verification: the first was the verification of the TCGA testing cohort, namely, the internal verification; the second was the verification of the whole TCGA cohort; the third was the clinical grouping verification, namely, subgroup survival analysis; and the last was the external verification of the ICGC cohort. Through the verification of the above four dimensions, we fully affirmed the prognostic value of the prognostic signature for HCC.

\section{Comparison of prognostic efficacy between the five-gene prognostic signature with other previously published prognostic models}

In recent years, new HCC prognostic signatures have been emerging. For example, the AUC values of the six- gene signature (SQSTM1, AHSA1, VNN2, SMG5, SRXN1, and GLS) constructed by Wenli et al. [30] were $0.761,0.681$, and 0.692 , respectively, in predicting the OS of TCGA cohort in 1, 3, and 5 years, and 0.681, 0.700 and 0.684 , respectively, in ICGC cohort. Another 14-gene signature (BIRC5, BUB1B, CCNB2, CDC45, DTL, GINS2, KIF23, KIF2C, MAD2L1, MCM4, OIP5, PLK4, PTTG1, and ZWINT) developed by Bo-Han Zhang et al. [31] were $0.714,0.740$, and 0.635 , respectively, in predicting the OS of TCGA cohort in 1, 3, and 5 years. The AUC values for our model in predicting the OS of patients in the TCGA cohort were $0.838,0.741$, and 0.692 respectively at 1,3 , and 5 years, and 0.746 , 0.778 , and 0.778 , respectively, in ICGC cohort. Therefore, our prognostic model is superior to the previously published prognostic models.

\section{The functions and pathways of the five-gene in HCC}

Sulfiredoxin 1 (SRXN1) acts as a key factor regulating the antioxidant response in eukaryotic cells, can resist oxidative stress injury in cells and has antioxidant protective effects on many diseases [32]. The findings of Lv [33] demonstrated that SRXN1 modulated ROS/p65/ BTG2 signaling, thereby stimulating $\mathrm{HCC}$ tumorigenesis and metastasis. Lactate dehydrogenase A (LDHA) serves as an important metabolic enzyme that is a member of the family of 2-hydroxy acid oxidoreductases and remarkably affects the anaerobic metabolism of cells [34]. Liu [35] reported that gankyrin upregulated LDHA expression, thereby increasing the consumption of glucose and glutamine and the production of lactate and glutamate in HCC, which might promote c-Myc-mediated tumorigenicity, metastasis, and drug resistance. The DP1 gene (TFDP1) acts as a heterodimerization partner for E2F family members of transcription factors, and E2F/ DP-1 regulates the expression of different cellular promoters, especially gene products that participate in the cell cycle [36]. TFDP1 has been identified as a c-Myctargeted gene by D Hunecke [37], which may promote hepatocyte transformation by changing cell cycle control, thus promoting the carcinogenic activity of c-Myc. Kohichiroh Yasui [36] also found that elevated TFDP1 expression may significantly affect HCC progression, as it promoted tumor cell growth. PPM1G serves as a nuclear-localized serine/threonine phosphatase that regulates chromatin remodeling, mRNA splicing, and DNA damage [38]. Khoronenkova [39] found that the ATMdependent protein phosphatase PPM1G dephosphorylated the ubiquitinase USP7 after ionizing radiation, resulting in the downregulation of USP7 and increased genomic instability, but its specific function has not been described previously in HCC. EIF2S1 promoted tumorigenesis by activating autophagy and enhancing tumor formation, enabling tumor cells to survive in a hypoxic 
and a low glucose microenvironment, making it an attractive target in Myc-driven cancer [40]. However, the function of EIF2S1 in HCC has not yet been reported.

\section{Future prospects}

This study identified and validated a prognostic model related to ARID1A mutations for the first time, which may be a reference to better understand the pathogenesis of HCC. In addition, the method remarkably lowers the sequencing costs, ensuring more routine and costeffective application of specific gene-based targeted sequencing, but for HCC patients who are mostly diagnosed by imaging modalities and treated with nonsurgical methods, the value of this model may be limited because our model needs to quantify the expression levels of eight specific genes in resected specimens [41]. As a retrospective study, we should acknowledge certain limitations to the study. It is necessary to conduct prospective and randomized controlled clinical studies that cover multiple centers and large sample sizes in the future. In addition, experimental analysis, including using a cell model and HCC tissues from the clinic, to validate the prediction is urgently needed.

\section{Conclusion}

Our research proposed a novel and robust approach for the prognostic risk classification of HCC patients, and this approach may provide new insights to improve the treatment strategy of HCC.

\section{Abbreviations}

HCC: Hepatocellular carcinoma; TCGA: The Cancer Genome Atlas; ICGC: International Cancer Genome Consortium; GSEA: Gene set enrichment analysis; ROC: Receiver operating characteristic; OS: Overall survival

\section{Supplementary Information}

The online version contains supplementary material available at https://doi. org/10.1186/s12957-021-02202-9. Additional file 1: Supplement material 1 The workflow chart of this
research.

Additional file 2: Supplement material 2 The clinico-pathological parameter of training and validation set.

Additional file 3: Supplement material 3 AJE language editing certificate.

Additional file 4: Supplement material 4 The detailed information of follow-up and stage for patients in the beginning of the Kaplan meier curve between 1-2 years.

Additional file 5: Supplement material 5 The relationship between the prognostic model and ARID1A (A) The heatmap of the prognostic model for TCGA (B) Kaplan-Meier survival analysis for the prognostic model applied on different ARID1A type in TCGA (C) The heatmap of the prognostic model for ICGC (D) Kaplan-Meier survival analysis for the prognostic model applied on different ARID1A type in ICGC.

\section{Authors' contributions}

Huibin Yang and Xin Li designed this study. Junyu Huo collected data, analyzed the data in this study, and interpreted the findings. Huibin Yang drafted the manuscript. Xin Li carried out data management and revised the manuscript. All authors reviewed the final version of the manuscript.

\section{Funding}

None

Availability of data and materials

The datasets analyzed for this study were obtained from The Cancer Genome Atlas (TCGA) (https://portal.gdc.cancer.gov/) and International Cancer Genome Consortium (ICGC) (https://icgc.org/)

\section{Declarations}

Competing Interest

The authors declare that they have no competing interests.

Ethics approval and consent to participate

Not applicable

\section{Consent for publication}

Not applicable

\section{Author details}

${ }^{1}$ Qingdao University, No. 308 Ningxia Road, Qingdao 266071, China.

${ }^{2}$ Department of Hepatobiliary and Pancreatic Surgery, The Affiliated Qingdao Municipal Hospital of Qingdao University, No.1 Jiaozhou Road, Shibei District, Qingdao City 266011, Shandong Province, China.

Received: 19 December 2020 Accepted: 18 March 2021

Published online: 26 March 2021

\section{References}

1. Shain AH, JRJPO P. The spectrum of SWI/SNF mutations, ubiquitous in human cancers. 2013;8(1):e55119.

2. Dallas PB, Pacchione S, Wilsker D, et al. The human SWI-SNF complex protein p270 is an ARID family member with non-sequence-specific DNA binding activity. 2000;20(9):3137-46

3. Wu R-C, Wang T-L, I-MJCb S. The emerging roles of ARID1A in tumor suppression. Therapy. 2014;15(6):655-64.

4. Ho L, Ronan JL, Wu J, et al. An embryonic stem cell chromatin remodeling complex, esBAF, is essential for embryonic stem cell self-renewal and pluripotency. 2009;106(13):5181-6.

5. Wu JN, CWJCd R. ARID1A mutations in cancer: another epigenetic tumor suppressor? 2013;3(1):35-43.

6. Samartzis EP, Noske A, Dedes K, Fink D, PJljoms I. ARID1A mutations and PI3K AKT pathway alterations in endometriosis and endometriosisassociated ovarian carcinomas. 2013;14(9):18824-49.

7. Luo B, Cheung HW, Subramanian A, et al. Highly parallel identification of essential genes in cancer cells. 2008;105(51):20380-5.

8. Huang J, Deng Q, Wang Q, et al. Exome sequencing of hepatitis B virusassociated hepatocellular carcinoma. 2012;44(10):1117-21.

9. He F, Li J, Xu J, et al. Decreased expression of ARID1A associates with poor prognosis and promotes metastases of hepatocellular carcinoma. 2015; 34(1):1-8.

10. Yim SY, Kang SH, Shin J-H, et al. Low ARID1A expression is associated with poor prognosis in hepatocellular carcinoma. 2020;9(9):2002.

11. Hu C, Li W, Tian F, et al. Arid1a regulates response to anti-angiogenic therapy in advanced hepatocellular carcinoma. 2018;68(3):465-75.

12. Bao S, Zhao H, Yuan J, et al. Computational identification of mutatorderived IncRNA signatures of genome instability for improving the clinical outcome of cancers: a case study in breast cancer. 2020;21(5):1742-55.

13. Somers EJCCMAJ. International agency for research on cancer. 1985;133(9): 845.

14. Cancer IAfRo. WHO classification of tumours of the digestive system: International Agency for Research on Cancer; 2019.

15. Rebouissou S, Nault JC. Advances in molecular classification and precision oncology in hepatocellular carcinoma. J hepatol. 2020;72(2):215-29. https:// doi.org/10.1016/j.jhep.2019.08.017. 
16. Zhang Q, Lou Y, Yang J, et al. Integrated multiomic analysis reveals comprehensive tumour heterogeneity and novel immunophenotypic classification in hepatocellular carcinomas. 2019;68(11).

17. Nakagawa H, Fujita M, Fujimoto A. Genome sequencing analysis of liver cancer for precision medicine. Semin Cancer Biol. 2019;55:120-7. https://doi. org/10.1016/j.semcancer.2018.03.004.

18. Shimada S, Mogushi K, Akiyama Y, et al. Comprehensive molecular and immunological characterization of hepatocellular carcinoma. 2019;40:457-70.

19. Schulze K, Nault J-C, AJJoh V. Genetic profiling of hepatocellular carcinoma using next-generation sequencing. 2016;65(5):1031-42.

20. Ally $A$, Balasundaram $M$, Carlsen $R$, et al. Comprehensive and integrative genomic characterization of hepatocellular carcinoma. 2017;169(7):1327-41 e23.

21. Sun X, Wang SC, Wei Y, Luo X, Jia Y, Li L, et al. Arid1a has contextdependent oncogenic and tumor suppressor functions in liver cancer. Cancer Cell. 2017;32(5):574-89 e6. https://doi.org/10.1016/j.ccell.2017.10.007.

22. Namjan A, Techasen A, Loilome W, Sa-Ngaimwibool P, Jusakul AJP. ARID1A alterations and their clinical significance in cholangiocarcinoma. 2020;8:e10464

23. Yang S-Z, Wang A-Q, Du J, et al. Low expression of ARID1A correlates with poor prognosis in intrahepatic cholangiocarcinoma. 2016;22(25):5814.

24. CVJC D. MYC on the path to cancer. 2012;149(1):22-35.

25. Albihn A, Johnsen Jl, MAJAicr H. MYC in oncogenesis and as a target for cancer therapies. 2010:107:163-224.

26. Soucek $L$, Whitfield J, Martins CP, et al. Modelling Myc inhibition as a cancer therapy. 2008;455(7213):679-83.

27. Stine ZE, Walton ZE, Altman BJ, Hsieh AL, CVJCd D. MYC, metabolism, and cancer. 2015;5(10):1024-39.

28. Moloney JN, Cotter TG. ROS signalling in the biology of cancer. Seminars in cell \& developmental biology. 2018: Elsevier;2018:50-64

29. Pelicano H, Carney D, PJDRU H. ROS stress in cancer cells and therapeutic implications. 2004;7(2):97-110.

30. Li W, Lu J, Ma Z, Zhao J, JJFig L. An integrated model based on a six-gene signature predicts overall survival in patients with hepatocellular carcinoma. 2020;10:1323.

31. Zhang B-H, Yang J, Jiang $L$, et al. Development and validation of a 14-gene signature for prognosis prediction in hepatocellular carcinoma. 2020;112(4): 2763-71.

32. Tahmasbpour Marzony E, Ghanei M, YJElr P. Oxidative stress and altered expression of peroxiredoxin genes family (PRDXS) and sulfiredoxin-1 (SRXN1) in human lung tissue following exposure to sulfur mustard. 2016; 42(4):217-26.

33. Lv X, Yu H, Zhang Q, et al. SRXN1 stimulates hepatocellular carcinoma tumorigenesis and metastasis through modulating ROS/p65/BTG2 signalling. 2020, SRXN1 stimulates hepatocellular carcinoma tumorigenesis and metastasis through modulating ROS/p65/BTG2 signalling.

34. Jafary F, Ganjalikhany MR, Moradi A, Hemati M, SJSr J. Novel peptide Inhibitors for Lactate Dehydrogenase A (LDHA): A survey to Inhibit LDHA Activity via Disruption of protein-protein Interaction. 2019;9(1):1-13.

35. Liu R, Li Y, Tian L, et al. Gankyrin drives metabolic reprogramming to promote tumorigenesis, metastasis and drug resistance through activating $\beta$-catenin/c-Myc signaling in human hepatocellular carcinoma. 2019;443:3446

36. Yasui K, Okamoto H, Arii S, JJJohg I. Association of over-expressed TFDP1 with progression of hepatocellular carcinomas. 2003;48(12):609-13.

37. Hunecke D, Spanel R, Länger F, Nam SW, JJTJop B. MYC-regulated genes involved in liver cell dysplasia identified in a transgenic model of liver cancer. 2012;228(4):520-33.

38. Foster WH, Langenbacher A, Gao C, Chen J, YJDD W. Nuclear phosphatase PPM1G in cellular survival and neural development. 2013;242(9):1101-9.

39. Khoronenkova SV, Dianova II, Ternette N, Kessler BM, Parsons JL, GLJMc D. ATM-dependent downregulation of USP7/HAUSP by PPM1G activates p53 response to DNA damage. 2012:45(6):801-13.

40. Dey S, Tameire F, CJA K. PERK-ing up autophagy during MYC-induced tumorigenesis. 2013;9(4):612-4.

41. Huo J, Wu L, Zang Y, et al. Eight-gene metabolic signature related with tumor-associated macrophages predicting overall survival for hepatocellular carcinoma. 2021:21(1):1-15.

\section{Publisher's Note}

Springer Nature remains neutral with regard to jurisdictional claims in published maps and institutional affiliations.

Ready to submit your research? Choose BMC and benefit from:

- fast, convenient online submission

- thorough peer review by experienced researchers in your field

- rapid publication on acceptance

- support for research data, including large and complex data types

- gold Open Access which fosters wider collaboration and increased citations

- maximum visibility for your research: over $100 \mathrm{M}$ website views per year

At BMC, research is always in progress.

Learn more biomedcentral.com/submissions 